\title{
High Kinetic Energy Ion Mobility Spectrometry (HiKE-IMS) at 40 mbar
}

\author{
Florian Schlottmann, Ansgar T. Kirk, Maria Allers, Alexander Bohnhorst, Stefan Zimmermann
}

Leibniz University Hannover, Institute of Electrical Engineering and Measurement Technology, Department of Sensors and Measurement Technology, Appelstr. 9A, 30167 Hannover, Germany

Abstract:

High Kinetic Energy Ion Mobility Spectrometers (HiKE-IMS) are usually operated at an absolute pressure of $20 \mathrm{mbar}$ reaching high reduced electric field strengths of up to $125 \mathrm{Td}$ for controlled reaction kinetics. This significantly increases the linear range and limits chemical cross sensitivities. Furthermore, HiKE-IMS enables the ionization of compounds normally not detectable in ambient pressure IMS, such as benzene, due to new reaction pathways and the inhibition of clustering reactions. In addition, HiKE-IMS allows the observation of additional orthogonal parameters related to an increased ion temperature such as fragmentation and field-dependent ion mobility, which may help to separate compounds that have similar ion mobility under low field conditions.

Aiming for a hand-held HiKE-IMS to carry its benefits into field applications, reducing size and power consumption of the vacuum system is necessary. In this work, we present a novel HiKE-IMS design entirely manufactured from standard printed circuit boards (PCB) and experimentally investigate the analytical performance in dependence of the operating pressure between 20 mbar and $40 \mathrm{mbar}$. Hereby, the limit of detection (LoD) for benzene in purified, dry air (1.4 ppmv water) improved from $7 \mathrm{ppb}_{\mathrm{v}}$ at $20 \mathrm{mbar}$ down to $1.8 \mathrm{ppb}_{\mathrm{v}}$ at $40 \mathrm{mbar}$. Furthermore, adding $0.9 \mathrm{ppm}_{\mathrm{v}}$ toluene the signal of the benzene $\mathrm{B}^{+}$peak decreases by only $2 \%$ at 40 mbar. Even in the presence of high relative humidity in the sample gas above $90 \%$ or toluene concentrations of up to $20 \mathrm{ppm} v$, the LoD for benzene just increases to $9 \mathrm{ppb}_{\mathrm{v}}$ at $40 \mathrm{mbar}$.

\section{Introduction}

Ion mobility spectrometry (IMS) is a well-known technique for fast online trace gas detection ${ }^{1,2}$. Providing limits of detection in the low $\mathrm{ppb}_{\mathrm{v}}$ (parts-per-billion by volume) or even $\mathrm{ppt}_{\mathrm{v}}$ (parts-pertrillion by volume) ${ }^{1}$ range within a second of measurement time, they are commonly used in safety and security applications for the fast detection of chemical warfare agents ${ }^{3,4}$, toxic industrial chemicals $^{5,6}$, drugs $s^{7,8}$ or explosives ${ }^{9-11}$. Ion mobility spectrometers separate ions by their motion through a neutral gas under the influence of an electric field. Classical ion mobility spectrometers in these applications are operated at ambient pressure of about 1000 mbar. Thus, they can be easily coupled to extremely efficient atmospheric pressure chemical ionization (APCI) sources. In these sources, electrons with high kinetic energy e.g. emitted from weak radioactive beta emitters or generated in corona discharges initiate a reaction cascade resulting in the ionization of analyte molecules. Initially, stable reactant ions are generated by ionizing the main constituents of the sample gas. Subsequently, these reactant ions react with analyte molecules to form product ions. Due to the large number of collisions at ambient pressure, this ionization method is very effective for substances amenable for this chemical ionization. Polar substances with high proton affinity are detected with high sensitivity. In contrast, nonpolar substances with low proton affinity are only detectable with great difficulty or not at all. Furthermore, the sensitivity is vastly decreasing with increasing water content of the introduced sample gas ${ }^{12,13}$. Additionally, IMS operated at ambient pressure suffers from a low linear range and strong matrix effects ${ }^{14-18}$, as the generated ion population does not represent the actual composition of the sample gas, but the thermodynamically controlled ion population after reaching the thermal equilibrium. 
To overcome these problems, in e.g. proton transfer reaction (PTR) or selected ion flow drift tube (SIDFT) mass spectrometry, the occurring chemical ionization processes are controlled in complex ionmolecule reactors operating at decreased pressures ${ }^{16}$. As described for example by Lindinger et al. ${ }^{19-}$ ${ }^{21}$ as well as Spanel and Smith ${ }^{22-25}$, in these reactors, reaction times in the order of $100 \mu \mathrm{s}$ to $1 \mathrm{~ms}$ result in a decrease in cross sensitivity as the ions are injected into the mass spectrometer before the thermal equilibrium establishes. Furthermore, the dissociation of ion-neutral clusters by high reduced electric field strengths $E / N$ enables a detection even of low proton affine and nonpolar substances. The reduced electric field strength $E / N$ is the ratio of electric field strength $E$ to neutral molecule density $N$, usually expressed in Townsend $\left(1 \mathrm{Td}=1 \cdot 10^{-21} \mathrm{Vm}^{2}\right)$. It is a measure for the energy an ion acquires in between to collisions.

Similar to PTR-MS or SIFDT-MS, High Kinetic Energy Ion Mobility Spectrometers (HiKE-IMS) ${ }^{26,27}$ ionize in a reaction region operated at decreased pressures around 20 mbar and reduced electric reaction field strengths $E / N$ up to $120 \mathrm{Td}$. However, instead of a mass spectrometer, an ion mobility spectrometer operated at the same pressure as the reaction region is used for ion separation and detection. Thus, compared to mass spectrometry neither ion transfer nor high vacuum components are needed. Due to the operation at decreased pressures and high reduced electric field strengths, the ion-molecule chemistry in the HiKE-IMS generally differs from that in classical ambient pressure IMS ${ }^{28}$. Nonetheless, the underlying ion-molecule reactions resulting in the formation of the observed ions in the HiKE-IMS are well known from literature. In particular, at thermal ion-molecule interaction energies, thermodynamic and kinetic data describing the occurring ion-molecule reactions have been published among others by Good ${ }^{29}$, Kebarle ${ }^{30-32}$ and Zhao ${ }^{33}$. Furthermore, extensive information is available from PTR- or SIFDT-MS studies concerning the reactions of different reactant ions with various analyte molecules ${ }^{34-42}$. High reduced electric field strengths $E / N$ of $125 \mathrm{Td}$ lead e.g. for hydronium $\left(\mathrm{H}_{3} \mathrm{O}^{+}\right)$to average kinetic energies of approx. $0.25 \mathrm{eV}^{43}$. As described above, the operation at decreased pressure and high reduced electric field strengths is particularly beneficial for two main reasons. First, the much shorter residence time of ions in the reaction region leads to a significant enhancement of the linear range and limited chemical cross sensitivities. Second, high reduced electric field strengths inhibit the formation of ion-neutral clusters and thus enable new ionization pathways to ionize e.g. low proton affine compounds not detectable by ambient pressure chemical ionization. For example, in a previous proof-of-concept study using HiKE-IMS ${ }^{27}$, a direct quantitative detection of the low proton affine substance benzene with $16 \mathrm{ppb}_{\vee}$ limits of detection in humid gas mixtures (3700 ppm $v$ water concentration) containing high proton affinity compounds was demonstrated. Another important benefit of HiKE-IMS is the possibility of changing the reduced electric field strength in the drift region independently of the reduced electric field in the reaction region in order to separate substances by their field-dependent ion mobility as known from FAIMS and DMS ${ }^{1,44-48}$ allowing for improved substance identification. Furthermore, high reduced electric field strength can lead to fragmentation ${ }^{28,49,50}$, also improving substance identification.

However, the HiKE-IMS presented in a previous work ${ }^{51}$ has an overall length of about $420 \mathrm{~mm}$. Furthermore, a membrane pump with a weight of $11 \mathrm{~kg}$ and a maximum electrical power consumption of $800 \mathrm{~W}$ is used. Weight and power consumption are both crucial factors for future mobile and handheld HiKE-IMS. Therefore, one development goal is to operate the HiKE-IMS at elevated pressures above 20 mbar allowing for smaller pumps with less power consumption. Thus, a new HiKE-IMS design was developed with an operating pressure of 40 mbar but still reaching high reduced electric field strengths of up to $105 \mathrm{Td}$. In the following, the effect of an increased pressure in HiKE-IMS on the analytical performance will be discussed. 


\section{Experimental}

As mentioned before, the maximum operating pressure is increased to 40 mbar whilst using the same voltage sources as in the previously presented setup ${ }^{51}$. Thus, it is possible to use the same isolated electronics for driving the corona discharge source and the ion gate. The maximum reduced electric field strength $\varepsilon_{\max }$ is given in Equation $1^{52}$ with the maximum voltage $U_{\max }$ and the maximum electric field $E_{\max }$ divided by the neutral molecule density $N$. Here, the generalized length $L$ is used. This equation is valid for both the reaction region and the drift region with their distinct lengths and voltage sources.

$$
\varepsilon_{\max }=\frac{E_{\max }}{N}=\frac{U_{\max }}{L \cdot N}
$$

Substituting the neutral molecule density $N$ from Equation 1 by using the ideal gas equation leads to Equation 2. As the thermodynamic temperature $T$ can only be varied in a small range due to used construction materials and experimental setup, and since the voltage $U_{\max }$ is limited by the voltage source, the length $L$ has to be decreased to reach the same maximum reduced electric field strength $\varepsilon_{\max }$ when increasing the pressure. Thus, doubling the operating pressure $p$ results directly in halving the length $L$.

$$
\varepsilon_{\max }=\frac{U_{\max } \cdot T \cdot k_{B}}{L \cdot p} \sim \frac{1}{L \cdot p}
$$

Consequently, the HiKE-IMS designed in this work and to be operated at $40 \mathrm{mbar}$ has a $150 \mathrm{~mm}$ drift region. For comparison, the length of the drift region of the previous presented HiKE-IMS ${ }^{51}$ operated at $20 \mathrm{mbar}$ is $306 \mathrm{~mm}$. The table of content graphic shows a photograph of the reaction region of the HiKE-IMS, which is entirely built from standard printed circuit boards similar to the work of Bohnhorst et al..$^{53}$.The default operating parameters are listed in Table 1. Before assembly, all parts are thoroughly cleaned in an ultrasonic bath and afterwards stored in a vacuum oven at $80^{\circ} \mathrm{C}$ for $24 \mathrm{~h}$ to prevent any outgassing during operation as this can significantly alter the ion population ${ }^{54}$. In Figure 1 , a schematic of the HiKE-IMS is shown.

Ions are formed in the reaction region by corona discharge ionization (Corona Needle, Agilent Technologies, USA) before they are separated in the drift region. The original HiKE-IMS published by Langejürgen et al. ${ }^{26,27}$ and Kirk et al. ${ }^{51}$ uses additive water fed to the corona discharge region to shift the reactant ions towards $\mathrm{H}^{+}\left(\mathrm{H}_{2} \mathrm{O}\right)_{m}$ and $\mathrm{NO}^{+}\left(\mathrm{H}_{2} \mathrm{O}\right)_{n}$ discriminating $\mathrm{O}_{2}{ }^{+}\left(\mathrm{H}_{2} \mathrm{O}\right)_{q}$ due to the formation of large water clusters promoting the conversion of $\mathrm{O}_{2}{ }^{+}\left(\mathrm{H}_{2} \mathrm{O}\right)_{q}$ to $\mathrm{H}^{+}\left(\mathrm{H}_{2} \mathrm{O}\right)_{m}$. In contrast, the presented device in this work does not use any additional water, thinking of later hand-held devices and an increased lifetime of drift gas filters. In both, the reaction and drift region, the reduced electric field strength can be independently increased up to $105 \mathrm{Td}$ by varying the corresponding voltages. If pressure fluctuations occur, the reaction and drift voltage are automatically adjusted by custom built software based on LabVIEW (National Instruments, U.S.) in order to assure a constant reduced electric field strength during data acquisition. lons are injected from the reaction region into the drift region through the new tristate ion gate. The new tristate ion gate consists of three grids and utilizes a certain switching sequence to avoid any mobility discrimination ${ }^{51}$. The gate opening time is $3 \mu \mathrm{s}$. Due to the relatively short drift times of less than $500 \mu \mathrm{s}$, the repetition rate for the ion injection is $2 \mathrm{kHz}$.

Drift and sample gas are directly fed into the HiKE-IMS from ambient pressure via flow restricting capillaries with $250 \mu \mathrm{m}$ inner diameter and adjusted lengths to provide gas flow rates of $10 \mathrm{ml} / \mathrm{min}$ (milliliter standard per minute, mass flow at reference conditions $20^{\circ} \mathrm{C}$ and $1013.25 \mathrm{mbar}$ ) for sample 
and $20 \mathrm{ml} / \mathrm{min}$ for drift gas. The drift gas purges the drift region and the reaction region and mixes within the reaction region with the sample gas. Purified, dry air (1.4 ppmv water) was used for both, the drift and sample gas. Additionally, the humidity of the sample gas can be varied over a wide range up to a relative humidity of $92.5 \%$ at the operating temperature of $25^{\circ} \mathrm{C}$. Low water concentrations in the order of ppmv are measured by a dew point sensor (Easidew Online, Michell Instruments, Germany) and high relative humidity above $1 \%$ with a moisture and temperature sensor (Testo 6352, Testo, Austria). All substances were purchased from Sigma Aldrich. In our experimental setup, the HiKE-IMS is evacuated via a membrane pump (MVP 040-2, Pfeiffer Vacuum, Germany). The pressure within the HiKE-IMS is monitored with a capacitive pressure gauge (CMR 362, Pfeiffer Vacuum, Germany). By adjusting the pumping rate via a throttle valve (EVN 116, Pfeiffer Vacuum, Germany), the pressure within the HiKE-IMS can be varied between 20 and 40 mbar. In this work, the reduced electric field strength was kept constant at $85 \mathrm{Td}$ in the reaction region and $105 \mathrm{Td}$ in the drift region.

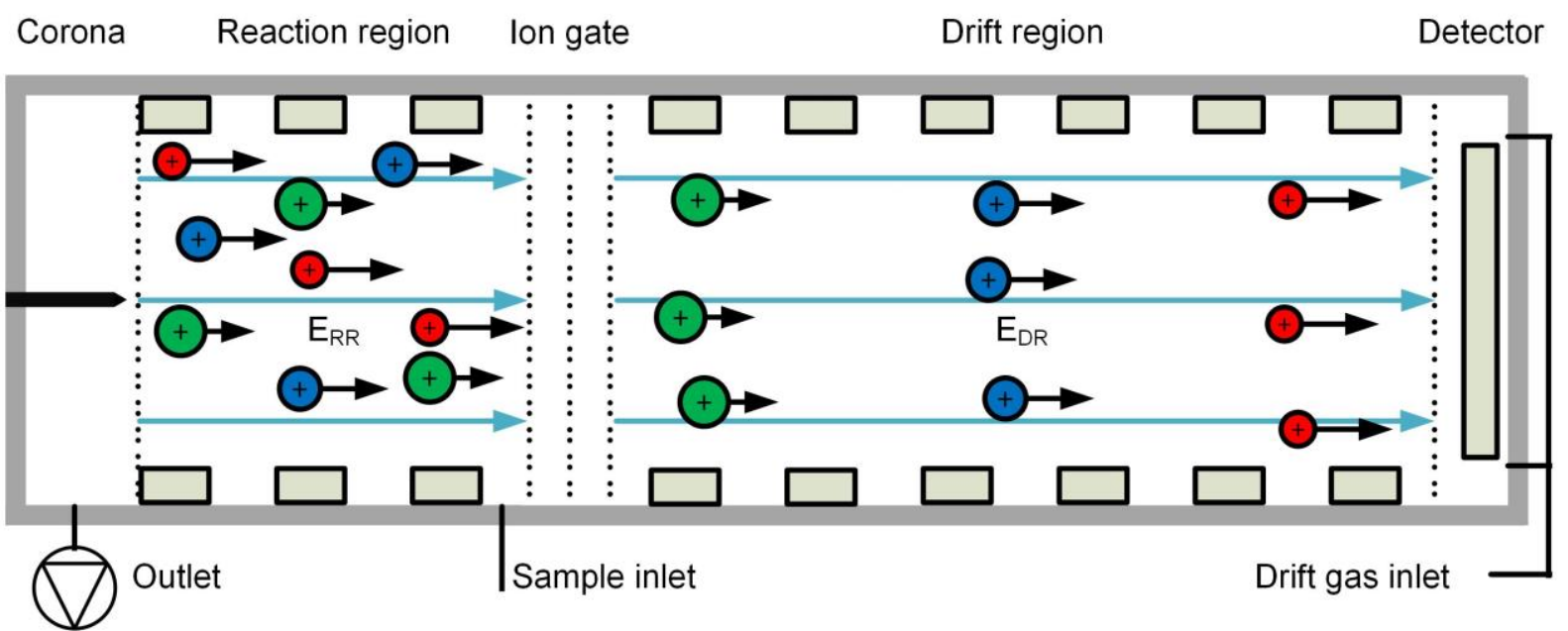

Figure 1: Schematic of the HiKE-IMS consisting of a corona discharge ionization source for generation of reactant ions, a reaction region where the reactant ions ionize analyte molecules in the sample gas, a tristate ion gate to chop the ion beam, a drift region in which ions are separated and a detector for detecting the ion current.

The electronics to drive the HiKE-IMS, such as the ion gate controller and the isolated voltage supply for the corona discharge source, are already reported $\mathrm{in}^{51}$. The ions discharge at a Faraday plate placed at the end of the drift region. Hereby, a current is measurable at the Faraday plate, which is amplified by a transimpedance amplifier with a bandwidth of $148 \mathrm{kHz}$ and a gain of $143 \mathrm{M} \Omega$ which was also designed and built at our institute ${ }^{55}$. A RTB2004, Rohde \& Schwarz, Germany, digitized the amplified ion mobility spectra. Additionally, a commercially available $20 \mathrm{kV}$ voltage source, HCP 14-20000, FuG Elektronik, Germany, was used for generating the drift voltage. At this stage of development, the setup is a laboratory grade demonstrator for testing the components, in this case, exploring effects of increasing operating pressure.

Table 1: Operational parameters of the HiKE-IMS used in this work

\begin{tabular}{|l|l|}
\hline Parameter & Value \\
\hline Reaction region length & $50 \mathrm{~mm}$ \\
\hline Drift region length & $150 \mathrm{~mm}$ \\
\hline $\begin{array}{l}\text { Overall length (including } \\
\text { corona ionization source and } \\
\text { connectors) }\end{array}$ & $250 \mathrm{~mm}$ \\
\hline $\begin{array}{l}\text { Inner Dimension of drift and } \\
\text { reaction region }\end{array}$ & $\begin{array}{l}20 \mathrm{~mm} \cdot 20 \mathrm{~mm}, \\
\text { rectangular shape }\end{array}$ \\
\hline Corona voltage & $1200-1550 \mathrm{~V}$ \\
\hline Reaction region voltage & $0-5 \mathrm{kV}$ \\
\hline
\end{tabular}




\begin{tabular}{|l|l|}
\hline Reaction region reduced field & $85 \mathrm{Td}$ \\
\hline Drift region voltage & $0-20 \mathrm{kV}$ \\
\hline Drift region reduced field & $105 \mathrm{Td}$ \\
\hline Injection time & $3 \mu \mathrm{s}$ \\
\hline Number of averages & 1024 \\
\hline Averaging time & $0.512 \mathrm{~s}$ \\
\hline Length of measured spectrum & $500 \mu \mathrm{s}$ \\
\hline Drift gas flow & $20 \mathrm{mls} /$ min \\
\hline Sample gas flow & $10 \mathrm{mls} /$ min \\
\hline $\begin{array}{l}\text { Dew point of } \\
\text { drift gas and sample gas }\end{array}$ & $-74{ }^{\circ} \mathrm{C}(1.4$ ppmv water \\
\hline Operating pressure & $20-40$ mbar \\
\hline Operating temperature & $25^{\circ} \mathrm{C}$ \\
\hline
\end{tabular}

\section{Results and Discussion}

The operating pressure has a profound impact on the analytical performance of the HiKE-IMS. One indicator for the performance of IMS is the resolving power $R_{p}$. The resolving power $R_{p}$ in ion mobility spectrometers exhibits an optimum between the regions where it is limited by diffusion and where it is limited by the initial ion packet width and amplifier broadening ${ }^{56,57}$. To achieve better limits of detection, ion mobility spectrometers for trace gas detection are usually operated in the latter region ${ }^{58,59}$. Thus, even at high reduced field strengths, effects such as increased diffusion due to ion heating just play a minor role ${ }^{51,60}$. The resulting resolving power equation when using the reduced field strength as the independent variable was previously derived by Kirk et al. ${ }^{51}$. Equation 3, which is taken from $^{51}$, shows also the dependence of the resolving power $R_{p}$ on the neutral molecule density $N$. Furthermore, constants like the charge of the ion $z \cdot e$, the Boltzman constant $k_{B}$, the neutral molecule density at standard conditions $N_{0}$ and the substance specific reduced ion mobility $K_{0}$ influence the different terms of the resolving power $R_{p}$. The temperature $T$ will not be varied in this paper. The same applies to the injection width $w_{i n j}$ and thus the resulting minimum temporal width $w_{\min }$ and the length of the drift region $L$. When operating at same reduced electric field strength $\varepsilon$, increasing the pressure will increase resolving power due to the reduced influence of diffusion ${ }^{51}$.

$$
R_{p}=\frac{1}{\sqrt{\left(\frac{w_{\min } \cdot K_{0} \cdot N_{0} \cdot \varepsilon}{L}\right)^{2}+\frac{16 \cdot T \cdot k_{B} \cdot \ln (2)}{Z \cdot e \cdot \varepsilon \cdot L \cdot N}}}
$$

Furthermore, the ion current $I_{\text {ion }}$ at the end of the reaction region of a HiKE-IMS with a corona discharge ionization source is shown in Equation 4, which was derived in ${ }^{61}$. The equation predicts a linear dependence between the ion current and pressure when the length $L$ and the reduced electric field strength $\varepsilon$ are kept constant. Thus, increasing operating pressure increases both, resolving power $R_{p}$ and ion current $I_{\text {ion }}$.

$$
I_{\text {ion }} \sim K_{0} \cdot \varepsilon^{2} \cdot \frac{N}{L}
$$

Since the total charge arriving at the detector is proportional to the ion current $I_{\text {ion }}$, the integral over time of the ion mobility spectrum, which gives the total charge, should be linear to the pressure. Figure 2 shows the measured total charge of the ion mobility spectrum of purified, dry air (1.4 ppm $\mathrm{V}$ water) with just the reactant ion peak present at different pressures. The predicted linearity 
dependence can be clearly seen. More generally, higher pressure leads to a larger number of reactant ions, which increases the linear range and the sensitivity of HiKE-IMS.

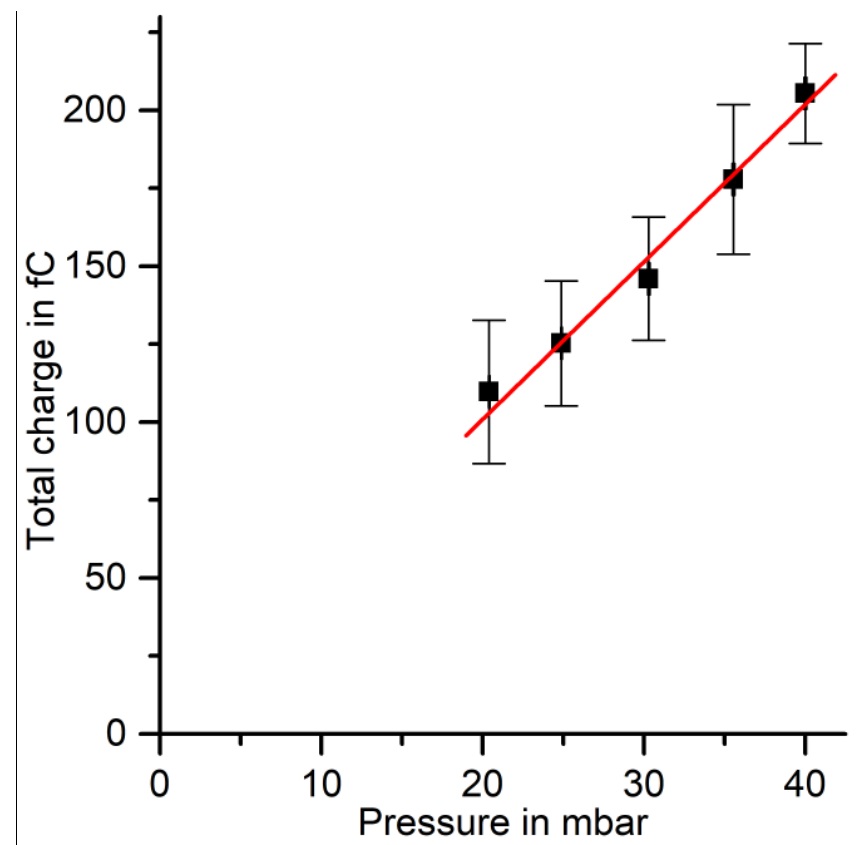

Figure 2: Total charge related to the ion mobility spectrum of purified, dry air (1.4 ppm $v$ water) with just the reactant ion peaks present at varying pressures. For the $y$-axis error bars $1.96 \sigma$ is used and $\sigma$ is determined from the total charge in eleven spectra recorded at each of the different pressures. Due to the high accuracy of the pressure gauge, $x$-axis error bars are hardly visible.

As shown in previous work by Langejürgen ${ }^{27}$, the HiKE-IMS is capable of detecting substances not detectable with conventional IMS. For example, benzene is a widely used aromatic chemical compound and a natural constituent of crude oil ${ }^{62}$ that is classified by the International Agency for Research on Cancer (IARC) as carcinogenic to humans ${ }^{63}$. Therefore, low legal exposure limits exists, e.g. $1 \mathrm{ppm}_{\mathrm{V}}$ for $15 \mathrm{~min}$ short time exposure and $100 \mathrm{ppb}_{\mathrm{V}}$ for $10 \mathrm{~h}$ long time exposure at workplaces given by the U.S. National Institute for Occupational Safety or Health (NIOSH) or $50 \mathrm{ppb}_{\vee}$ for eight hours given by the European Chemicals Agency (ECHA). Thus, the LoD for benzene of the HiKE-IMS should be significantly below these values.

However, benzene is a nonpolar molecule and can easily lose charge to other substances. Furthermore, with a low proton affinity of just $750.4 \mathrm{~kJ} / \mathrm{mol}^{64}$ it is not likely to be ionized in conventional IMS operated around 1000 mbar as large water clusters having much higher proton affinity than benzene constitute the reactant ions. Even worse, concentrations of several ppb toluene, a substance likely to appear together with benzene e.g. in crude oil, completely suppresses any benzene related signal due to the higher proton affinity of toluene of $784 \mathrm{~kJ} / \mathrm{mol}^{64}$. Since all chemical ionization processes depend on pressure, the reactant ion population crucial for ionizing benzene is investigated whilst varying the pressure.

Figure 3 (left) shows the measured ion mobility spectra of purified, dry air (1.4 ppmv water) with just the reactant ions present for different operating pressures. The presented spectra consist of three reactant ion species. By coupling a HiKE-IMS to a mass spectrometer (MS), the underlying ion species have been identified as $\mathrm{NO}^{+}, \mathrm{H}_{3} \mathrm{O}^{+}$and $\mathrm{O}_{2}^{+28}$. When varying the pressure in 5 mbar steps from $20 \mathrm{mbar}$ to $40 \mathrm{mbar}$, the applied corona voltage needs to be adjusted, due to the varying neutral molecule density; otherwise, the corona discharge would extinguish. Corona voltage was varied between $1200 \mathrm{~V}$ at $20 \mathrm{mbar}$ and $1535 \mathrm{~V}$ at $40 \mathrm{mbar}$. The reactant ion spectra in Figure 3 (left) show different variations in peak height of the three reactant ion species with increasing pressure. Nevertheless, the total charge is linearly increasing with pressure, as already shown in Figure 2. 
Intensities of $\mathrm{NO}^{+}$and $\mathrm{H}_{3} \mathrm{O}^{+}$significantly increase with pressure, while the intensity of $\mathrm{O}_{2}{ }^{+}$slightly decreases. This is due to the increasing number of collisions with pressure and therefore a higher possibility for $\mathrm{O}_{2}{ }^{+}$ions losing charge to molecules forming more stable ions. Figure 3 (right) shows the relative abundance of each reactant ion species charge on the total charge of the spectra in Figure 3 (left). Looking at the nearly constant relative abundance of $\mathrm{NO}^{+}$, such ions seem to be chemically unaffected by increasing pressure besides their absolute increase directly imposed by increasing pressure. In contrast, with increasing pressure the relative abundance of $\mathrm{O}_{2}{ }^{+}$is decreasing in the same manner as the relative abundance of $\mathrm{H}_{3} \mathrm{O}^{+}$increases. The increasing number of collisions with increasing pressure in a reaction region of constant length leads to an increasing conversion of $\mathrm{O}_{2}{ }^{+}$to $\mathrm{H}_{3} \mathrm{O}^{+}$reactant ions. In summary, higher pressures lead to a higher absolute number of $\mathrm{NO}^{+}$and $\mathrm{H}_{3} \mathrm{O}^{+}$ while the absolute number of $\mathrm{O}_{2}{ }^{+}$remains nearly constant. This leads to new reaction pathways, such as the charge transfer from $\mathrm{NO}^{+}$and $\mathrm{O}_{2}{ }^{+}$to benzene giving $\mathrm{B}^{+}$.
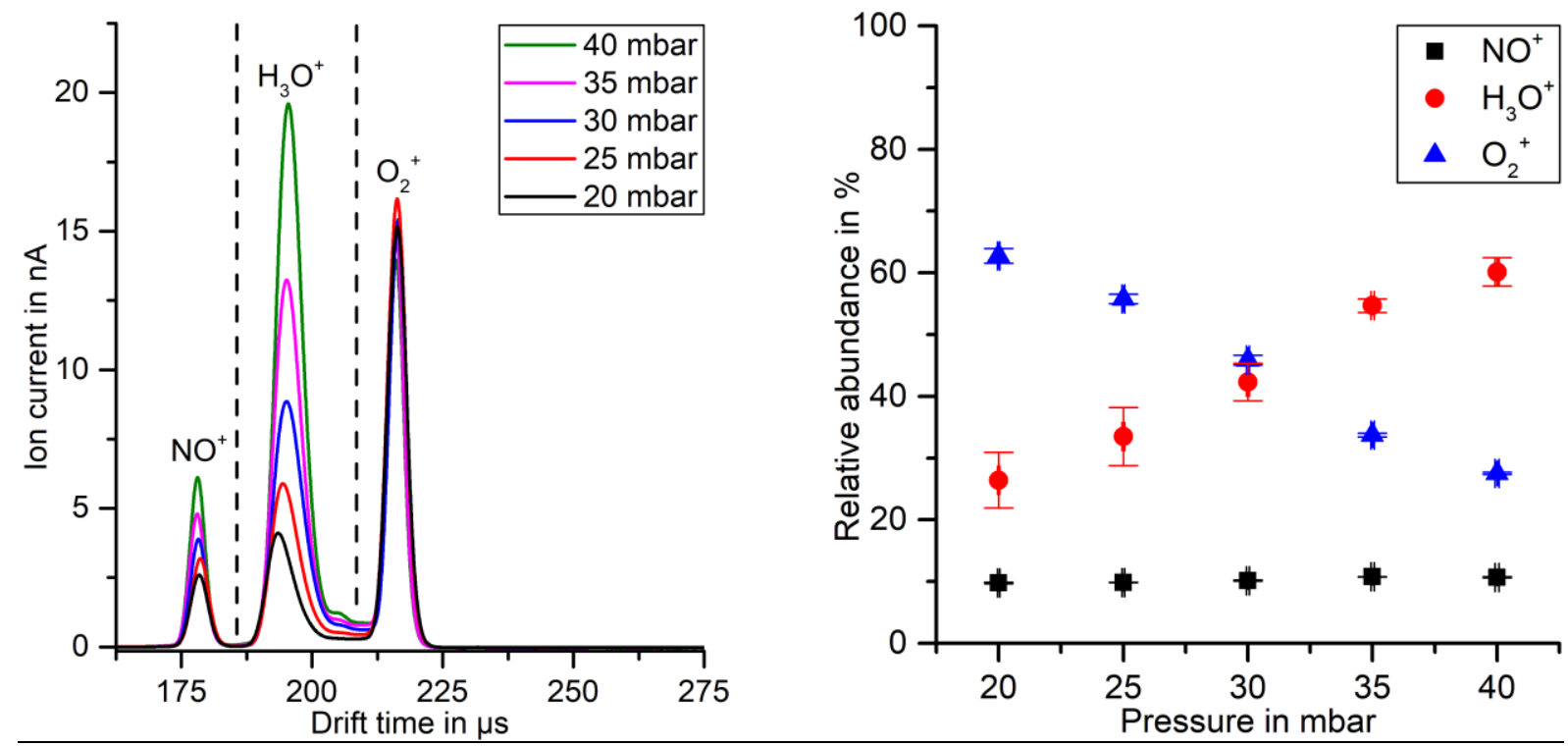

Figure 3: Reactant ion spectra of purified, dry air (1.4 ppm $\mathrm{p}_{V}$ water) at different pressures, reduced ion mobility of $\mathrm{NO}^{+}=3.04 \mathrm{~cm}^{2} / \mathrm{Vs}, \mathrm{H}_{3} \mathrm{O}^{+}=2.80 \mathrm{~cm}^{2} / \mathrm{Vs}$ and $\mathrm{O}_{2}{ }^{+}=2.50 \mathrm{~cm}^{2} / \mathrm{Vs}$ (left). Relative abundance of reactant ions charge on the total charge of the spectra in Figure 3 (left) over pressure (right). For the y-axis error bars 1.96 $\sigma$ is used and $\sigma$ is determined from the respective charge in eleven spectra recorded at each of the different pressures. Due to the high accuracy of the pressure gauge, $x$-axis error bars are hardly visible.

Having analyzed the pressure-dependent changes of the reactant ions available for ionizing benzene, the ionization of benzene should be considered next. Therefore, the simple charge transfer reaction with the rate constant $k_{R B}$ between a reactant ion $\mathrm{R}^{+}$, which either can be $\mathrm{NO}^{+}$or $\mathrm{O}_{2}{ }^{+}$, and the analyte benzene $B$ is considered in Equation 5 .

$$
R^{+}+B \stackrel{k_{R B}}{\longrightarrow} R+B^{+}
$$

In order to evaluate the time-dependent change of $B^{+}$in the reaction region of the HiKE-IMS, reaction 5 and its corresponding reaction rate constant can be expressed by an ordinary differential equation. Since the neutral reaction partner benzene is present in vast excess, the reaction is considered to be pseudo-first order with respect to the ionic species. The solution of the resulting pseudo-first-order differential equation provides the number of the single charged Benzene ions per volume $\left[\mathrm{B}^{+}\right]$for short reaction times $\Delta t$, as derived for example in ${ }^{65}$ and shown in Equation 6. 
The number of reactant ions per volume $\left[R^{+}\right]_{0}$ at the beginning of the reaction is proportional to the ion current $I_{i o n}$ and the number of neutral benzene molecules per volume $[B]$ is proportional to the neutral molecule density $N$. The reaction time $\Delta t$ is the drift time through the reaction region as known from standard ion mobility spectrometry ${ }^{1}$ leading to a quadratic increase of $\left[B^{+}\right]$with pressure as given in Equation 7. These considerations are only valid as long as no concurrent reactions exist or the number of $\left[R^{+}\right]_{0}$ is high enough.

$$
\left[B^{+}\right] \sim I_{i o n} \cdot N \cdot \frac{L_{R R}}{v_{d}} \sim \frac{\varepsilon^{2} N}{L_{R R}} \cdot N \cdot \frac{L_{R R}}{K_{0} \cdot \varepsilon} \sim \varepsilon \cdot N^{2} \sim N^{2}
$$

Summarizing the model for product ion generation, it is obvious from the given equations that the population of reactant ions linearly increases with pressure and results in a quadratic growth of product ions, thus, a significant increase in HiKE-IMS sensitivity. To verify the above relations, the ionization of benzene at a constant concentration of $750 \mathrm{ppb}_{v}$ is considered. Figure 4 (left) shows the recorded HiKE-IMS spectra in purified, dry air (1.4 ppmv water) for different operating pressures from 20 mbar to 40 mbar. Apart from the reactant ion species, two product ions attributed to benzene can be observed. Using the HiKE-IMS-MS coupling presented $\mathrm{in}^{28}$, these product ions have been identified as the single charged benzene $B^{+}$and the protonated benzene $B H^{+}$. As described before, $B^{+}$results from a direct charge transfer reaction with $\mathrm{NO}^{+}$and $\mathrm{O}_{2}{ }^{+} . \mathrm{BH}^{+}$is formed by proton transfer reaction with $\mathrm{H}_{3} \mathrm{O}^{+}$due to the lower proton affinity of the water molecule $\left(691 \mathrm{~kJ} / \mathrm{mol}^{64}\right)$ compared to that of benzene $\left(750.4 \mathrm{~kJ} / \mathrm{mol}^{64}\right) . B^{+}$is the dominant product ion of benzene with increasing number of molecules per volume for higher pressures as predicted by Equations 6 and 7. Figure 4 (right) shows the $B^{+}$ion current in dependence of pressure. As it can be seen, the $B^{+}$peak height grows in a quadratic fashion.
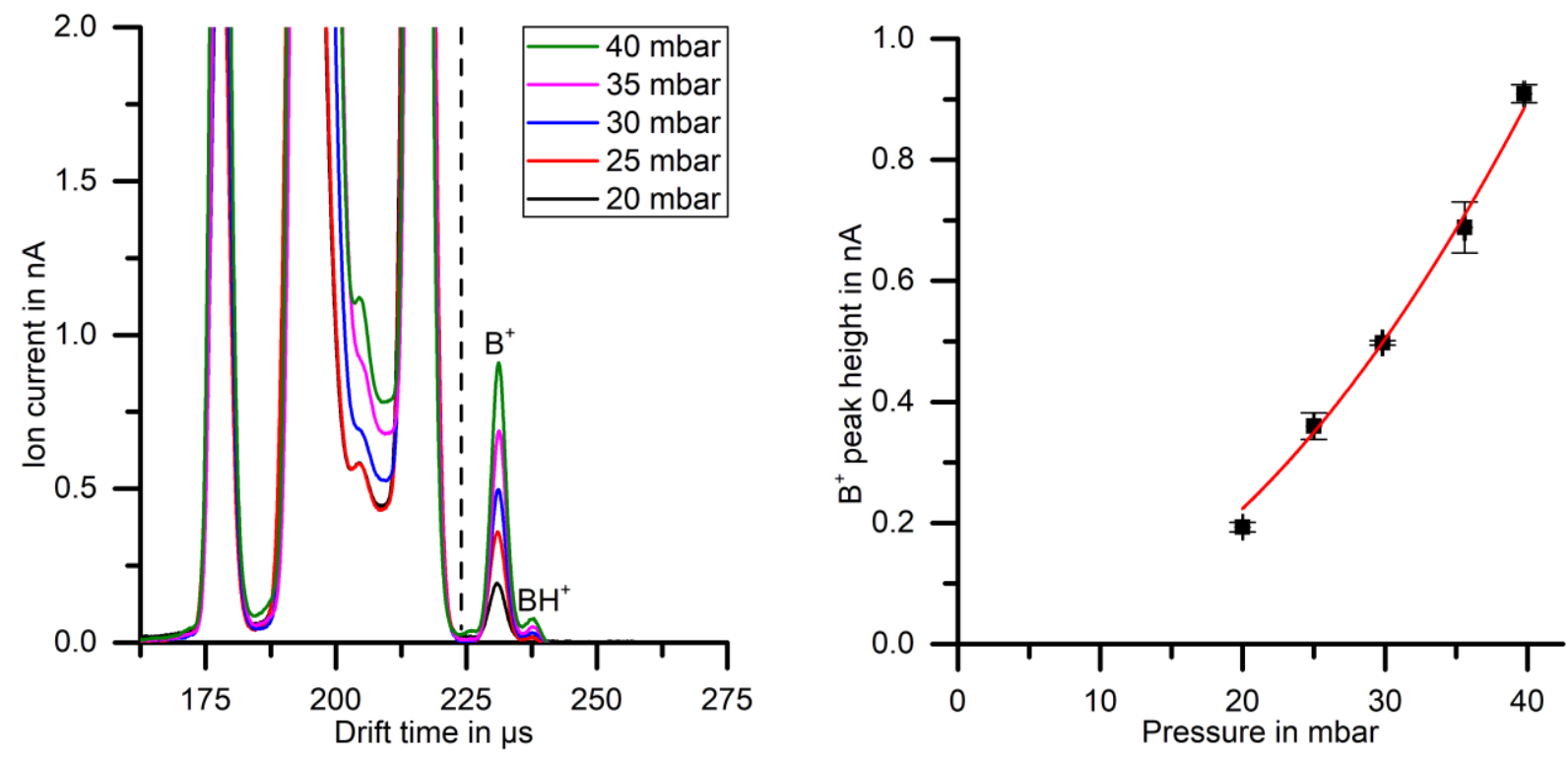

Figure 4: IMS spectra of $750 \mathrm{ppb}_{V}$ benzene in purified, dry air (1.4 ppm $v$ water) at different pressures, reduced ion mobility of $B^{+}=2.33 \mathrm{~cm}^{2} / \mathrm{Vs}$ and $\mathrm{BH}^{+} 2.26 \mathrm{~cm}^{2} / \mathrm{Vs}$ (left). Peak height of $B^{+}$over pressure and quadratic fit with no offset (right). For the $y$-axis error bars $1.96 \sigma$ is used and $\sigma$ is determined from the amplitude in at least ten spectra recorded at each of the different pressures. Due to the high accuracy of the pressure gauge, $x$-axis error bars are hardly visible.

To emphasize the major benefit of increasing pressure and the associated quadratic increase of product ions, limits of detection (LoD) were measured for different pressures. Here, the LoD is defined 
as the $B^{+}$signal being larger than thrice the noise $\sigma$ at given averages. Therefore, an orange line representing the $3 \sigma$ threshold is plotted in Figure 5 . As stated above, the peak height of benzene $B^{+}$ increases in a quadratic fashion with pressure. Likewise, the LoD improves from 6.4 to $1.8 \mathrm{ppb}_{v}$ with pressure increase from $20 \mathrm{mbar}$ to $40 \mathrm{mbar}$. The LoD has been calculated with $0.512 \mathrm{~s}$ of averaging.

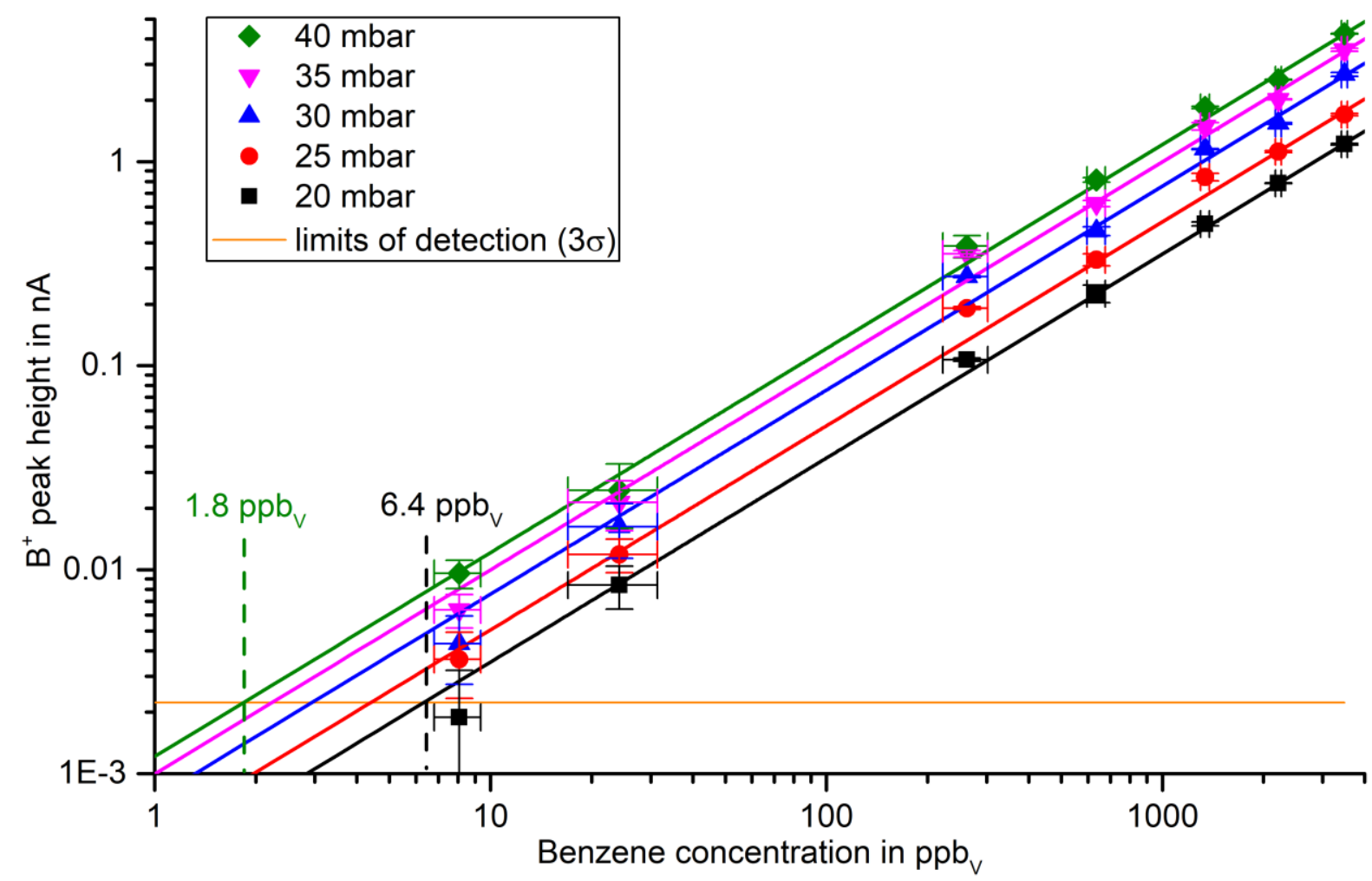

Figure 5: $B^{+}$peak height over concentration at different pressures and detecton limits of benzene in purified, dry air (1.4 ppm water). For the $y$-axis error bars, $1.96 \sigma$ is used and $\sigma$ is determined from the amplitude in at least ten spectra recorded at each of the different concentrations. In addition, for the concentration error bars on the $x$-axis calculations of the relative error were performed. At 24 ppb benzene concentration, another flow controller is switched on, significantly enlarging the error.

In the following, the effect of toluene and water on the benzene signal is investigated at an operation pressure of 40 mbar. In conventional IMS operated at ambient pressure of about 1000 mbar, water is a major interferent due to the increasing cluster size of the protonated water clusters leading to an increased proton affinity, e.g. of $808 \mathrm{~kJ} / \mathrm{mol}^{64}$ for the water cluster $\left(\mathrm{H}_{2} \mathrm{O}\right)_{2}$. Thus, low proton affine substances, such as benzene with a proton affinity of $750.4 \mathrm{~kJ} / \mathrm{mol}$, cannot be ionized under humid conditions. In HiKE-IMS the situation is quite different for various reasons. Figure 6 shows the recorded ion mobility spectra of $750 \mathrm{ppb}_{\vee}$ benzene in purified air with increasing relative humidity. Obviously, increasing humidity in the sample gas leads to a significant change of the reactant ion population. The intensity of the $\mathrm{NO}^{+}$peak slightly increases with increasing sample gas humidity. In contrast, the abundance of $\mathrm{O}_{2}{ }^{+}$is heavily affected by the relative humidity and decreases with increasing humidity. At high relative humidity, all $\mathrm{O}_{2}{ }^{+}$are converted to $\mathrm{H}_{3} \mathrm{O}^{+66}$. Thus, at high humidity, the formation of $\mathrm{B}^{+}$via the charge transfer reaction with $\mathrm{O}_{2}{ }^{+}$is not possible and the ionization via $\mathrm{NO}^{+}$is the only possible ionization path. Consequently, losing one path for ionization, the $B^{+}$signal is reduced to $0.166 \mathrm{nA}$ at $92.5 \%$ relative humidity as shown in Figure 6 (left). Comparing this peak height to the peak height of $750 \mathrm{ppb}_{\vee} B^{+}$in purified, dry air (1.4 ppm $\vee$ water), leads to a decrease by a factor of five, which would lead to an increase of the LoD of $B^{+}$to 9 ppbv. 

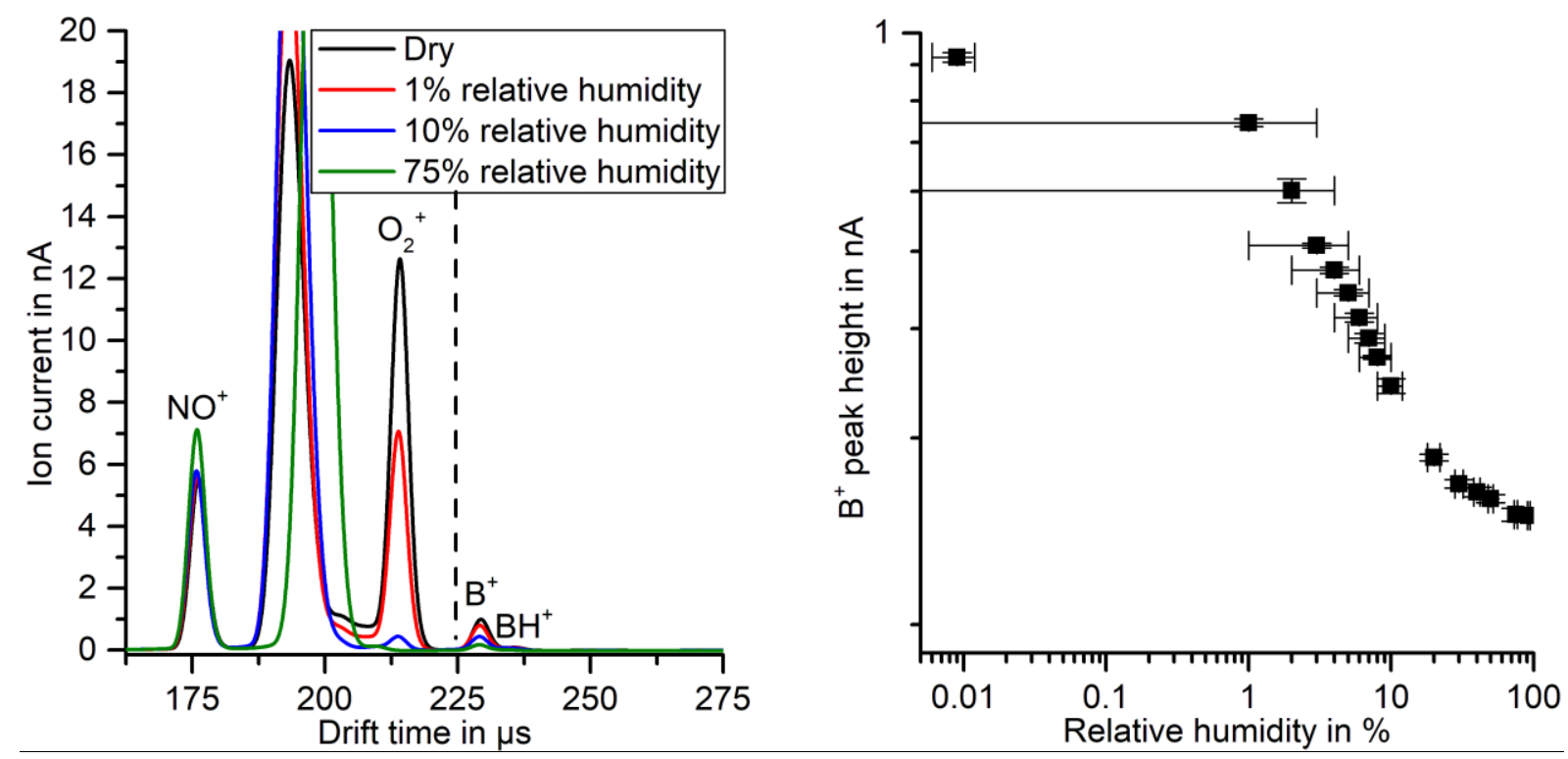

Figure 6: Ion mobility spectra of 750 ppbv benzene in purified air with changing relative humidity at 40 mbar (left). Peak height of $B^{+}$at increasing relative humidity (right). For the $y$-axis error bars $1.96 \sigma$ is used and $\sigma$ is determined from the amplitude in at least ten spectra recorded. For the $x$-axis error bars the percentage error of the relative humidity measuring device is used. The lowest humidity at $0.009 \%$ is calculated from the given dew point and its error.

In addition to water, other substances chemically interfere with benzene in conventional IMS operated at ambient pressure, e.g. other aromatic hydrocarbons such as toluene and xylene. Both toluene and xylene have a higher proton affinities of $784 \mathrm{~kJ} / \mathrm{mol}$ and $796 \mathrm{~kJ} / \mathrm{mol}{ }^{64}$ than benzene of $750.4 \mathrm{~kJ} / \mathrm{mol}$, resulting in a chemical suppression of the benzene signal even for very low concentrations of toluene and benzene. Thus, the effect of an increasing toluene concentration on the benzene signal is investigated next, see Figure 7 (left). The concentration of benzene is constant at $750 \mathrm{ppbv}$. Similar to benzene, toluene can be ionized via different ionization pathways and forms two peaks, a direct ionized, single charged $T^{+}$and a protonated $\mathrm{TH}^{+}$. While $0.9 \mathrm{ppm} \mathrm{v}$ toluene has no visible effect on the $B^{+}$peak height, $5 \mathrm{ppm} v$ toluene reduces the $B^{+}$peak to $72 \%$ of its initial height. This is a significantly reduced interference compared to conventional IMS. In Figure 7 (right), the peak height of $B^{+}$is plotted over the toluene concentration whilst increasing the toluene concentration from zero ppm $\mathrm{v}$ up to $22 \mathrm{ppm}_{\mathrm{v}}$. The $B^{+}$peak height is decreasing from $0.8 \mathrm{nA}$ until it settles at a minimum of $0.2 \mathrm{nA}$ at a toluene concentration of $22 \mathrm{ppm} v$. At the used parameters, a significant cross sensitivity of toluene with respect to benzene exists. By increasing the pressure and therefore the number of collisions, such increasing cross sensitivity is expected. Nonetheless, in contrast to ambient pressure IMS, the HiKEIMS operated at 40 mbar clearly outplays conventional IMS regarding the detection of benzene in the presence of toluene and water. By looking at the $B^{+}$peak height change in the presence of $22 \mathrm{ppm} v$ toluene, a limit of detection of $8 \mathrm{ppm}_{\mathrm{v}}$ benzene would still be achieved in toluene rich environments within $0.512 \mathrm{~s}$ of averaging. 

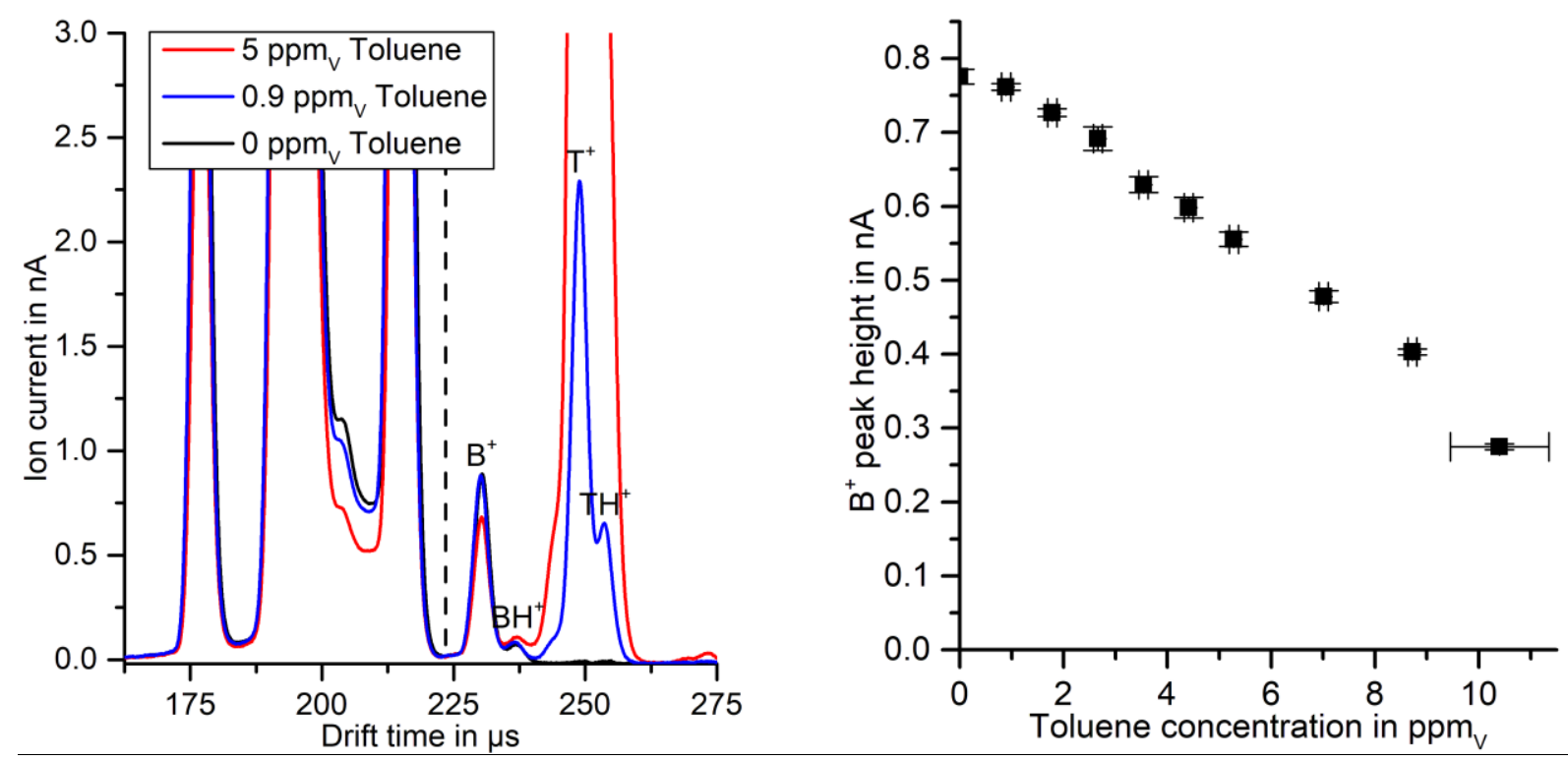

Figure 7: Ion mobility spectra at different toluene concentrations and constant benzene concentration of 750 ppb in purified, dry air (1.4 ppm $v$ water) at $40 \mathrm{mbar}, T^{+} 2.18 \mathrm{~cm}^{2} / \mathrm{Vs}$ and $\mathrm{TH}^{+} 2.14 \mathrm{~cm}^{2} / \mathrm{Vs}$ (left). Peak height of $B^{+}$and $T^{+}$at increasing toluene concentration. For the $y$-axis error bars, $1.96 \sigma$ is used and $\sigma$ is determined from the amplitude in at least ten spectra recorded. In addition, for the concentration error bars on the x-axis calculations of the relative error were performed. At $10.3 \mathrm{ppm}_{V}$ toluene concentration, another flow controller is switched on, significantly enlarging the error.

\section{Conclusion}

In this paper, we present a miniaturized, just $250 \mathrm{~mm}$ long HiKE-IMS entirely built from printed circuit boards, operated at a maximum pressure of $40 \mathrm{mbar}$, and still reaching reduced electric field strengths in the reaction and drift region of up to $105 \mathrm{Td}$. With miniaturized electronics and peripherals, construction of a field portable, briefcase-sized HiKE-IMS is therefore feasible. By increasing pressure at constant reduced electric field strength, the reactant ion current increases, enlarging the number of possible reaction partners for analyte molecules. This results in a quadratic increase in analyte ion generation, here demonstrated for benzene. Furthermore, two important cross-sensitivities, water and toluene, have been investigated. As expected, cross-sensitivities increase with increasing pressure but benzene is clearly detectable with a LoD of $9 \mathrm{ppb}_{\mathrm{v}}$ in less than a second even at very high concentrations of toluene up to $22 \mathrm{ppm}_{\mathrm{v}}$ and relative humidity up to $92.5 \%$. Thus, the presented HiKEIMS has a five times lower LoD for benzene than the required value given by the European Chemicals Agency (ECHA) for long-term exposures ( $50 \mathrm{ppb}$ ).

\section{Acknowledgement}

This work is supported by the German Federal Ministry of Education and Research (BMBF) under the grant $13 \mathrm{~N} 14469$

\section{References}

(1) Eiceman, G.A., Karpas, Z., Hill, H.H.: Ion mobility spectrometry, 3rd ed., CRC Press, Boca Raton (2013)

(2) Borsdorf, H., Mayer, T., Zarejousheghani, M., Eiceman, G.A.: Recent Developments in lon Mobility Spectrometry, Appl. Spectrosc. Rev., 46, 472-521, (2011)

(3) Puton, J., Namieśnik, J.: Ion mobility spectrometry, TrAC, Trends Anal. Chem., 85, 10-20, (2016)

(4) Mäkinen, M.A., Anttalainen, O.A., Sillanpää, Mika E T: Ion mobility spectrometry and its applications in detection of chemical warfare agents, Anal. Chem., 82, 9594-9600, (2010) 
(5) Mayer, T., Borsdorf, H.: Which parameters influence the quantitative determination of halogenated substances? A summary of systematic investigations, Int. J. Ion Mobil. Spec., (2015)

(6) Gaik, U., Sillanpää, M., Witkiewicz, Z., Puton, J.: Nitrogen oxides as dopants for the detection of aromatic compounds with ion mobility spectrometry, Anal. Bioanal. Chem., 409, 3223-3231, (2017)

(7) Zaknoun, H., Binette, M.-J., Tam, M.: Analyzing fentanyl and fentanyl analogues by ion mobility spectrometry, Int. J. Ion Mobil. Spec., 58, 366, (2019)

(8) Armenta, S., La Guardia, M. de, Alcalà, M., Blanco, M., Perez-Alfonso, C., Galipienso, N.: Ion mobility spectrometry evaluation of cocaine occupational exposure in forensic laboratories, Talanta, 130, 251-258, (2014)

(9) R.G. Ewing, D.A. Atkinson, G.A. Eiceman, G.J. Ewing: A critical review of ion mobility spectrometry for the detection of explosives and explosive related compounds, Talanta, 54, 515-529, (2001)

(10) Mäkinen, M., Nousiainen, M., Sillanpää, M.: Ion spectrometric detection technologies for ultratraces of explosives: a review, Mass Spectrom Rev, 30, 940-973, (2011)

(11) Buryakov, I.A.: Detection of explosives by ion mobility spectrometry, J Anal Chem, 66, 674-694, (2011)

(12) Safaei, Z., Willy, T.J., Eiceman, G.A., Stone, J.A., Sillanpää, M.: Quantitative response in ion mobility spectrometry with atmospheric pressure chemical ionization in positive polarity as a function of moisture and temperature, Anal. Chim. Acta, 1092, 144-150, (2019)

(13) Kuklya, A., Uteschil, F., Kerpen, K., Marks, R., Telgheder, U.: Effect of the humidity on analysis of aromatic compounds with planar differential ion mobility spectrometry, Int. J. Ion Mobil. Spec., 18, 67-75, (2015)

(14) Eiceman, G.A., Vandiver, V.J.: Charge-exchange in binary mixtures of polycyclic aromatic hydrocarbons using photoionization-ion mobility spectrometry, Anal. Chem., 58, 2331-2335, (1986)

(15) Puton, J., Holopainen, S.I., Mäkinen, M.A., Sillanpää, Mika E T: Quantitative response of IMS detector for mixtures containing two active components, Anal. Chem., 84, 9131-9138, (2012)

(16) Puton, J., Augustyniak, D., Perycz, U., Witkiewicz, Z.: Conservation of dimer peak intensity in ion mobility spectrometers with ketone-doped carrier gas, Int. J. Mass Spectrom., 373, 43-49, (2014)

(17) Vandiver, V.J., Leasure, C.S., Eiceman, G.A.: Proton affinity equilibria for polycyclic aromatic hydrocarbons at atmospheric pressure in ion mobility spectrometry, Int. J. Mass Spectrom. Ion Process., 66, 223-238, (1985)

(18) Eiceman, G.A.: Advances in Ion Mobility Spectrometry, 22, 471-490, (1991)

(19) Hansel, A., Jordan, A., Warneke, C., Holzinger, R., Wisthaler, A., Lindinger, W.: Proton-transferreaction mass spectrometry (PTR-MS), Plasma Sources Sci. Technol., 8, 332-336, (1999)

(20) Lagg, A., Taucher, J., Hansel, A., Lindinger, W.: Applications of proton transfer reactions to gas analysis, Int. J. Mass Spectrom. Ion Process., 134, 55-66, (1994)

(21) Lindinger, W., Hansel, A.: Analysis of trace gases at $\mathrm{ppb}$ levels by proton transfer reaction mass spectrometry (PTR-MS), Plasma Sources Sci. Technol., 6, 111-117, (1997)

(22) Spesyvyi, A., Smith, D., Španěl, P.: Selected Ion Flow-Drift Tube Mass Spectrometry: Quantification of Volatile Compounds in Air and Breath, Anal. Chem., (2015)

(23) Španěl, P., Spesyvyi, A., Smith, D.: Electrostatic Switching and Selection of $\mathrm{H} 3 \mathrm{O}+, \mathrm{NO}+$, and $\mathrm{O} 2+\bullet$ Reagent lons for Selected Ion Flow-Drift Tube Mass Spectrometric Analyses of Air and Breath, Anal. Chem., (2019)

(24) Spesyvyi, A., Smith, D., Španěl, P.: Ion chemistry at elevated ion-molecule interaction energies in a selected ion flow-drift tube, Phys. Chem. Chem. Phys., 19, 31714-31723, (2017) 
(25) Smith, D., Spanel, P.: Selected ion flow tube mass spectrometry (SIFT-MS) for on-line trace gas analysis, Mass Spectrom Rev, 24, 661-700, (2005)

(26) Langejürgen, J., Allers, M., Oermann, J., Kirk, A.T., Zimmermann, S.: High kinetic energy ion mobility spectrometer: quantitative analysis of gas mixtures with ion mobility spectrometry, Anal. Chem., 86, 7023-7032, (2014)

(27) Langejürgen, J., Allers, M., Oermann, J., Kirk, A.T., Zimmermann, S.: Quantitative detection of benzene in toluene- and xylene-rich atmospheres using high-kinetic-energy ion mobility spectrometry (IMS), Anal. Chem., 86, 11841-11846, (2014)

(28) Allers, M., Kirk, A.T., Roßbitzky, N. von, Erdogdu, D., Hillen, R., Wissdorf, W., Benter, T., Zimmermann, S.: Analyzing Positive Reactant lons in High Kinetic Energy lon Mobility Spectrometry (HiKE-IMS) by HiKE-IMS-MS, J. Am. Soc. Mass Spectrom., 31, 812-821, (2020)

(29) Good, A.: Ion-Molecule Reactions in Pure Nitrogen and Nitrogen Containing Traces of Water at Total Pressures 0.5-4 torr. Kinetics of Clustering Reactions Forming $\mathrm{H}+(\mathrm{H} 2 \mathrm{O}) \mathrm{n}$, J. Chem. Phys., 52, 212, (1970)

(30) French, M.A., Hills, L.P., Kebarle, P.: Kinetics and Temperature Dependence of the Hydration of $\mathrm{NO}+$ in the Gas Phase, Can. J. Chem., 51, 456-461, (1973)

(31) Kebarle, P., Searles, S.K., Zolla, A., Scarborough, J., Arshadi, M.: Solvation of the hydrogen ion by water molecules in the gas phase. Heats and entropies of solvation of individual reactions. $\mathrm{H}+(\mathrm{H} 2 \mathrm{O}) \mathrm{n}-1+\mathrm{H} 2 \mathrm{O}$.fwdarw. H+(H2O)n, J. Am. Chem. Soc., 89, 6393-6399, (1967)

(32) Lau, Y.K., Ikuta, S., Kebarle, P.: Thermodynamics and kinetics of the gas-phase reactions $\mathrm{H} 3 \mathrm{O}+(\mathrm{H} 2 \mathrm{O}) \mathrm{n}-1+$ water $=\mathrm{H} 3 \mathrm{O}+(\mathrm{H} 2 \mathrm{O}) \mathrm{n}, \mathrm{J}$. Am. Chem. Soc., 104, 1462-1469, (1982)

(33) Zhao, J., Zhang, R.: Proton transfer reaction rate constants between hydronium ion ( $\mathrm{H} 3 \mathrm{O}+$ ) and volatile organic compounds, 38, 2177-2185, (2004)

(34) McFarland, M., Albritton, D.L., Fehsenfeld, F.C., Ferguson, E.E., Schmeltekopf, A.L.: Flow-drift technique for ion mobility and ion-molecule reaction rate constant measurements. II. Positive ion reactions of $\mathrm{N}+\mathrm{O}+$ and $\mathrm{H} 2+$ with $\mathrm{O} 2$ and $\mathrm{O}+$ with $\mathrm{N} 2$ from thermal to $\sim 2 \mathrm{eV}, \mathrm{J}$. Chem. Phys., 59, 6620-6628, (1973)

(35) Španěl, P., Smith, D.: Selected ion flow tube studies of the reactions of $\mathrm{H} 3 \mathrm{O}+$, NO+, and $\mathrm{O} 2+$ with several aromatic and aliphatic hydrocarbons, Int. J. Mass Spectrom., 181, 1-10, (1998)

(36) Španěl, P., Žabka, J., Zymak, I., Smith, D.: Selected ion flow tube study of the reactions of H3 O+ and $\mathrm{NO}+$ with a series of primary alcohols in the presence of water vapour in support of selected ion flow tube mass spectrometry, RCM, 31, 437-446, (2017)

(37) Spanel, P., Smith, D.: SIFT studies of the reactions of $\mathrm{H} 3 \mathrm{O}+$, NO+ and $\mathrm{O} 2+$ with a series of alcohols, Int. J. Mass Spectrom. Ion Process., 167-168, 375-388, (1997)

(38) Španěl, P., Smith, D.: SIFT studies of the reactions of $\mathrm{H} 3 \mathrm{O}+$, NO+ and $\mathrm{O} 2+$ with several ethers, Int. J. Mass Spectrom. Ion Process., 172, 239-247, (1998)

(39) Španěl, P., Ji, Y., Smith, D.: SIFT studies of the reactions of $\mathrm{H} 3 \mathrm{O}+$, NO+ and O2+ with a series of aldehydes and ketones, Int. J. Mass Spectrom. Ion Process., 165-166, 25-37, (1997)

(40) Kemper, P.R., Bowers, M.T.: A hybrid double-focusing mass spectrometer-High-pressure drift reaction cell to study thermal energy reactions of mass-selected ions, J. Am. Soc. Mass Spectrom., 1, 197-207, (1990)

(41) Wyttenbach, T., Bowers, M.T.: Modern Mass Spectrometry: Gas-Phase Conformations: The Ion Mobility/lon Chromatography Method, Springer-Verlag, Berlin, Heidelberg, New York (2003)

(42) McFarland, M., Albritton, D.L., Fehsenfeld, F.C., Ferguson, E.E., Schmeltekopf, A.L.: Flow-drift technique for ion mobility and ion-molecule reaction rate constant measurements. I. Apparatus and mobility measurements, J. Chem. Phys., 59, 6610, (1973)

(43) Hansel, A., Jordan, A., Holzinger, R., Prazeller, P., Vogel, W., Lindinger, W.: Proton transfer reaction mass spectrometry: on-line trace gas analysis at the ppb level, Int. J. Mass Spectrom. Ion Process., 149-150, 609-619, (1995) 
(44) Shvartsburg, A.A., Bryskiewicz, T., Purves, R.W., Tang, K., Guevremont, R., Smith, R.D.: Field asymmetric waveform ion mobility spectrometry studies of proteins: Dipole alignment in ion mobility spectrometry?, J. Phys. Chem. B, 110, 21966-21980, (2006)

(45) Shvartsburg, A.A.: Differential mobility spectrometry: FAIMS and beyond, CRC; Taylor \& Francis [distributor], Boca Raton, Fla., London (2008)

(46) Wannier, G.H.: Motion of Gaseous Ions in Strong Electric Fields, Bell Syst. Tech. J., 32, 170-254, (1953)

(47) Wyttenbach, T., Helden, G., Batka, J.J., Carlat, D., Bowers, M.T.: Effect of the long-range potential on ion mobility measurements, J. Am. Soc. Mass Spectrom., 8, 275-282, (1997)

(48) Viehland, L.A., Mason, E.A.: Gaseous ion mobility and diffusion in electric fields of arbitrary strength, 110, 287-328, (1978)

(49) Bohnhorst, A., Kirk, A.T., Yin, Y., Zimmermann, S.: Ion fragmentation and filtering by alpha function in ion mobility spectrometry for improved compound differentiation, Anal. Chem., 91, 8941-8947, (2019)

(50) Shokri, H., Vuki, M., Gardner, B., Niu, H.-C.W., Chiluwal, U., Gurung, B.K., Emery, D.B., Eiceman, G.A.: Reactive Tandem Ion Mobility Spectrometry with Electric Field Fragmentation of Alcohols at Ambient Pressure, Anal. Chem., (2019)

(51) Kirk, A.T., Grube, D., Kobelt, T., Wendt, C., Zimmermann, S.: A High Resolution High Kinetic Energy Ion Mobility Spectrometer Based on a Low-Discrimination Tristate Ion Shutter, Anal. Chem., 90, 5603-5611, (2018)

(52) Mason, E.A., McDaniel, E.W.: Transport Properties of lons in Gases, Wiley-VCH Verlag GmbH \& Co. KGaA, Weinheim, FRG (1988)

(53) Bohnhorst, A., Kirk, A.T., Zimmermann, S.: Simulation aided design of a low cost ion mobility spectrometer based on printed circuit boards, Int. J. Ion Mobil. Spec., 19, 167-174, (2016)

(54) Maißer, A., Thomas, J.M., Larriba-Andaluz, C., He, S., Hogan, C.J.: The mass-mobility distributions of ions produced by a Po-210 source in air, J. Aerosol Sci., 90, 36-50, (2015)

(55) Cochems, P., Kirk, A.T., Zimmermann, S.: In-circuit-measurement of parasitic elements in high gain high bandwidth low noise transimpedance amplifiers, Rev. Sci. Instrum., 85, 124703, (2014)

(56) Siems, W.F., Wu, C., Tarver, E.E., Hill, Herbert H. Jr., Larsen, P.R., McMinn, D.G.: Measuring the Resolving Power of Ion Mobility Spectrometers, Anal. Chem., 66, 4195-4201, (1994)

(57) Kirk, A.T., Allers, M., Cochems, P., Langejürgen, J., Zimmermann, S.: A compact high resolution ion mobility spectrometer for fast trace gas analysis, Analyst, 138, 5200-5207, (2013)

(58) Kanu, A.B., Gribb, M.M., Hill, H.H.: Predicting optimal resolving power for ambient pressure ion mobility spectrometry, Anal. Chem., 80, 6610-6619, (2008)

(59) Kirk, A.T., Zimmermann, S.: An analytical model for the optimum drift voltage of drift tube ion mobility spectrometers with respect to resolving power and detection limits, Int. J. Ion Mobil. Spec., 18, 129-135, (2015)

(60) Verbeck, G.F., Ruotolo, B.T., Gillig, K.J., Russell, D.H.: Resolution equations for high-field ion mobility, J. Am. Soc. Mass Spectrom., 15, 1320-1324, (2004)

(61) Kirk, A.T., Kobelt, T., Spehlbrink, H., Zimmermann, S.: A Simple Analytical Model for Predicting the Detectable Ion Current in Ion Mobility Spectrometry Using Corona Discharge Ionization Sources, J. Am. Soc. Mass Spectrom., (2018)

(62) World Health Organization: Occupational Exposures in Petroleum Refining; Crude Oil and Major Petroleum Fuels, IARC Monogr Eval Carcinog Risks Hum Suppl, (1989)

(63) World Health Organization: Overall evaluations of carcinogenicity: an updating of IARC Monographs volumes 1 to 42, IARC Monogr Eval Carcinog Risks Hum Suppl, 7, 1-440, (1987)

(64) Hunter, E. P. L., Lias, S.G.: Evaluated Gas Phase Basicities and Proton Affinities of Molecules: An Update, J. Phys. Chem. Ref. Data, 27, 413-656, (1998) 
(65) Gouw, J. de, Warneke, C., Karl, T., Eerdekens, G., van der Veen, Carina, and Fall, R.: Sensitivity and specificity of atmospheric trace gas detection by proton-transfer-reaction mass spectrometry, Int. J. Mass Spectrom., 223-224, 365-382, (2003)

(66) Fehsenfeld, F.C., Mosesman, M., Ferguson, E.E.: Ion-Molecule Reactions in an $\mathrm{O} 2$ +- 20 System, J. Chem. Phys., 55, 2115-2120, (1971) 